\title{
Dense Molecular Gas at High Redshift: First Detection of Emission from $\mathrm{HCO}^{+}$
}

\author{
D. A. Riechers ${ }^{1}$, F. Walter ${ }^{1}$, C. L. Carilli ${ }^{2}$, A. Weiss ${ }^{3}$, F. Bertoldi ${ }^{4}$, \\ K. M. Menten ${ }^{3}$, K. K. Knudsen ${ }^{1}$, and P. $\operatorname{Cox}^{5}$ \\ ${ }^{1}$ Max-Planck-Institut für Astronomie, Königstuhl 17, Heidelberg, D-69117, Germany \\ ${ }^{2}$ National Radio Astronomy Observatory, PO Box O, Socorro, NM 87801, USA \\ ${ }^{3}$ Max-Planck-Institut für Radioastronomie, Auf dem Hügel 69, Bonn, D-53121, Germany \\ ${ }^{4}$ Argelander-Institut für Astronomie, Auf dem Hügel 71, Bonn, D-53121, Germany \\ ${ }^{5}$ Institut d.RadioAstronomie Millimétrique, 300 R.d.l.Piscine, 38406 St.Martin d'Héres, France
}

\begin{abstract}
Using the Very Large Array (VLA), we have detected the $\mathrm{HCO}^{+}(1-0)$ emission line towards the Cloverleaf quasar $(z=2.56$; Riechers et al. 2006). This is the first detection of ionized molecular gas emission at high redshift $(z>2)$. $\mathrm{HCO}^{+}$emission is a star formation indicator similar to $\mathrm{HCN}$, tracing dense molecular hydrogen gas within star-forming molecular clouds. We find a $\mathrm{HCO}^{+} / \mathrm{CO}$ luminosity ratio of 0.08 and a $\mathrm{HCO}^{+} / \mathrm{HCN}$ luminosity ratio of 0.8 for the Cloverleaf. These ratios fall within the scatter of the same relationships found for low $-z$ star-forming galaxies. However, $\mathrm{a} \mathrm{HCO}^{+} / \mathrm{HCN}$ luminosity ratio close to unity would not be expected for the Cloverleaf if the recently suggested relation between this ratio and the farinfrared luminosity (Graciá-Carpio et al. 2006) were to hold. We conclude that a ratio between $\mathrm{HCO}^{+}$and $\mathrm{HCN}$ luminosity close to 1 is likely due to the fact that the emission from both lines is optically thick and thermalized and emerges from dense regions of similar volumes. We conclude that $\mathrm{HCO}^{+}$is potentially a good tracer for dense molecular gas at high redshift.
\end{abstract}

Keywords. galaxies: active, starburst, formation, high redshift, cosmology: observations, radio lines: galaxies

\section{Acknowledgements}

D. R. acknowledges support from the Deutsche Forschungsgemeinschaft (DFG) Priority Programme 1177. C. C. acknowledges support from the Max-Planck-Gesellschaft and the Alexander von Humboldt-Stiftung through the Max-Planck-Forschungspreis.

\section{References}

Graciá-Carpio, J., García-Burillo, S., Planesas, P., \& Colina, L. 2006, ApJ 640, L135

Riechers, D. A., Walter, F., Carilli, C. L., et al. 2006, ApJ 645, L13 